\title{
Influence of Crosslinker Identity and Position on Gas-Phase Dissociation of Lys-Lys Crosslinked Peptides
}

\author{
Sara P. Gaucher, Masood Z. Hadi, and Malin M. Young \\ Sandia National Laboratories, Livermore, California, USA
}

\begin{abstract}
A systematic study of the dissociation patterns of crosslinked peptides analyzed by tandem mass spectrometry is reported. A series of 11-mer peptides was designed around either a polyalanine or polyglycine scaffold with arginine at the $\mathrm{C}$ terminus. One or two lysine residues were included at various locations within the peptides to effect inter- or intra-molecular crosslinking, respectively. Crosslinked species were generated with four commonly used amine-specific chemical crosslinking reagents: disuccinimidyl suberate (DSS), disuccinimidyl tartarate (DST), dithiobis(succinimidylpropionate) (DSP), and disuccinimidyl glutarate (DSG). The influence of precursor charge state, location of crosslink, and specific crosslinking reagent on the MS/MS dissociation pattern was examined. Observed trends in the dissociation patterns obtained for these species will allow for improvements to software used in the automated interpretation of crosslinked peptide MS/MS data. (J Am Soc Mass Spectrom 2006, 17, 395-405) (c 2006 American Society for Mass Spectrometry
\end{abstract}

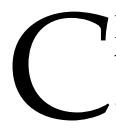
hemical crosslinking has been demonstrated to be an important tool for probing protein structure, particularly where a crystal structure is not yet available [1-7]. Crosslinking chemistry also is used in the analysis of protein complexes, where it has been applied to identify binding partners [8-15], characterize the interface of interacting subunits [16-20], and stabilize transient interactions [21,22]. There are several possible experimental approaches, many of which rely on specifically identifying and characterizing the crosslinked species by mass spectrometry. Both LCMS and LCMS/MS have been used. A current challenge for MS/MS studies is that little data exists to aid interpretation of crosslinked peptide MS/MS, unlike the wealth of data available for model peptide MS/MS.

To improve the methodology, work has been done to more closely scrutinize the specificity of crosslinker reactivity [23, 24], develop software for data analysis [25-28], and develop and test crosslinkers with certain properties such as affinity tags for product purification or stable isotope labels [29-34]. Interpretation of the peptide sequences from a crosslinked species still remains a challenge, however. Much "unexpected reactivity" has been found, thus MS/MS confirmation can be important.

Although much fundamental research has been done on gas-phase dissociation of protonated and cationized peptides [35-40], only limited MS/MS studies have

Published online January 27, 2006

Address reprint requests to Dr. S. P. Gaucher, Biosystems Research, Sandia National Laboratories, P.O. Box 969, MS 9291, Livermore, CA 94551-0969, USA. E-mail: spgauch@sandia.gov been performed on crosslinked peptide species [25, 41]. Given that the ability to determine crosslinked peptide sequence is a key component of many experimental approaches incorporating chemical crosslinking, it would be of great advantage to know more about how the crosslinked peptides dissociate in MS/MS so that database search methods and de novo analysis can be optimized for these spectra.

In this manuscript we report initial findings of ongoing work to systematically study the dissociation patterns of crosslinked peptides. In the current study we report results on the influence of precursor charge state, location of crosslink, and specific crosslinker used for inter and intra molecularly crosslinked Lys-Lys species.

\section{Experimental}

\section{Materials}

Peptides were custom synthesized by SynPep (Dublin, $\mathrm{CA}$ ) each with a blocked amino terminus (N-acetyl) and provided at $>98 \%$ purity as determined by HPLC. The crosslinkers disuccinimidyl suberate (DSS), disuccinimidyl tartarate (DST), dithiobis(succinimidylpropionate) (DSP), and disuccinimidyl glutarate (DSG) were purchased from Pierce (Rockford, IL). Triethylamine (TEA) was purchased from FisherBiotech (Fair Lawn, NJ). Milli-Q deionized water was used. Methanol and dimethyl sulfoxide (DMSO) were purchased from Burdick and Jackson (Muskegon, MI). Formic acid was purchased from EM Science (Darmstadt, Germany). 
1 Ac-AAKAAAAAAAR

$\underline{2}$ Ac-AAAAKAAAAAR

$\underline{3}$ Ac-AAAAAAAAKAR

4 Ac-GGGGKGGGGGR

\section{$\underline{5}$ Ac-AAAAKAKAAAR \\ 6 Ac-AAKAAAAAKAR}

Figure 1. Custom synthesized peptides used in crosslinking experiments.

\section{Crosslinking Reactions}

Stock solutions $(10 \mathrm{mg} / \mathrm{mL})$ of each peptide and crosslinker were prepared in water and DMSO, respectively. The crosslinker stock solution was always freshly prepared. Reactions were prepared by allowing 200 nmol total peptide to react with $200 \mathrm{nmol}$ total crosslinker. Total reaction volume was $50 \mu \mathrm{L}$ in $50 \mathrm{mM}$ TEA/carbonate buffer, $\mathrm{pH}$ 8.4. Reactions were incubated at RT for $30 \mathrm{~min}$, and stored at $4{ }^{\circ} \mathrm{C}$ until MS analysis. Peptides and crosslinkers used in these reactions are shown in Figures 1 and 2.

\section{Mass Spectrometry}

Samples were prepared for mass spectrometry by diluting $1 \mu \mathrm{L}$ of a given reaction to $1000 \mu \mathrm{L}$ in $50 \%$ aqueous<smiles>O=C(CCCC(=O)ON1C(=O)CCC1=O)ON1C(=O)CCC1=O</smiles><smiles>O=C(ON1C(=O)CCC1=O)C(O)C(O)C(=O)ON1C(=O)CCC1=O</smiles><smiles>O=C(CCCCCCC(=O)ON1C(=O)CCC1=O)ON1C(=O)CCC1=O</smiles><smiles>O=C(CCSSCCC(=O)ON1C(=O)CCC1=O)ON1C(=O)CCC1=O</smiles>

Figure 2. Commercially available crosslinkers used.

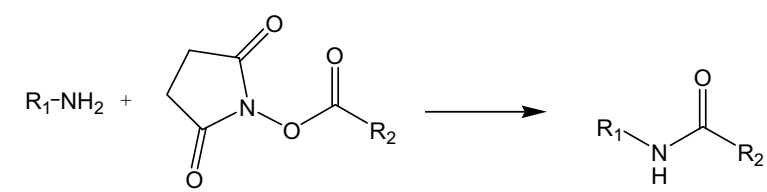

Scheme 1

methanol containing $0.1 \%$ formic acid. For MALDI TOF analysis, $1 \mu \mathrm{L}$ of diluted sample was mixed 1:1 with $\alpha$-cyano-4-hydroxy-cinnamic acid (Agilent, Palo Alto, CA) using the dried droplet method. Spectra were acquired on an Applied Biosystems Voyager DE PRO in positive reflectron mode, and close external calibration was used. For ESI MS, diluted samples were directly infused at $2 \mu \mathrm{L} / \mathrm{min}$ into a Micromass QTOF running Masslynx 4.0. Spectra were acquired in positive ion "v"-mode. Collision energy was tuned to deplete the precursor ion to $\sim 5 \%$ of the original isolated intensity. Spectra were also acquired using identical collision energies for each series of isomeric precursor ions for comparison. Spectra were deconvoluted using the MaxEnt3 algorithm provided with the Masslynx software.

\section{Results and Discussion}

\section{Experimental Design and Crosslinked Products}

The goal of this study was to systematically and specifically probe the influence of crosslinker identity and position on dissociation of crosslinked peptides in the absence of other factors such as amino acid composition. Thus, a series of peptides designed around either a polyalanine or polyglycine scaffold was synthesized as shown in Figure 1. However, to incorporate two "real world" features into the study, each peptide contained 11 total amino acids with arginine (Arg, R) at the C terminus to approximate the average size and Cterminal characteristic of peptides produced from a tryptic digest, a common enzymatic digest protocol. One or two lysine (Lys, K) residues were included at various locations within the peptide to effect inter- or intra-molecular crosslinking, respectively. The $\mathrm{N}$ terminus of each peptide was blocked by acetylation so that only the desired Lys-Lys crosslinks would be produced. The four chemical crosslinking reagents used in this study are shown in Figure 2 and the reaction is diagramed in Scheme $\mathbf{1}$.

Several different products arise from the reaction between a chemical crosslinking reagent and a peptide/ protein. Nomenclature for these product types has already been described [25] and will be used here. Briefly, an intramolecular crosslink is termed Type 1, while an intermolecular crosslink is termed Type 2 . The peptides within the Type 2 crosslink are differentiated by $\alpha$ and $\beta$ with the $\alpha$ chain having the longer chain length or greater mass. A common side reaction product observed when working with homobifunctional NHS ester crosslinkers is a dead-end crosslink where 


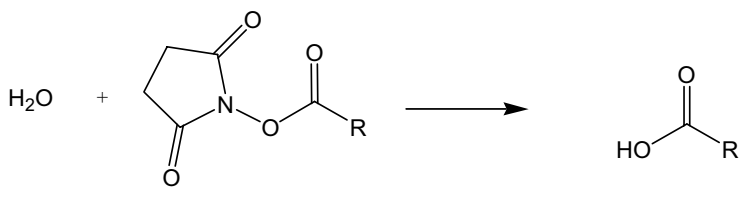

Scheme 2

only one amide bond has been formed between a peptide and one end of the crosslinking reagent. The other NHS ester moiety has undergone hydrolysis and is no longer reactive. This hydrolysis reaction is outlined in Scheme 2. These deadend "crosslinked" products have been termed Type 0 .

The products formed in each of the reactions examined here were initially screened by MALDI TOF. Spectra from the crude reaction mixtures of peptides 2 and 4 with DSS and of peptide 5 with DSS are shown in Supplementary Material Figure 1a and b, respectively (which can be found in the electronic version of this article). The products observed are representative of the reactions carried out with peptides 1-4 to generate Type 2 products (Supplementary Material Figure 1a) and with peptides 5 and 6 to generate Type 1 products (Supplementary Material Figure 1b). In both cases shown here, some starting material remains $(\mathrm{m} / \mathrm{z}$ 984.5, $77 \%$ relative abundance and $m / z$ 858.4, 64\% RA for peptides 2 and 4 reaction; $m / z$ 1041.7, 76\% RA for peptide 5 reaction). The relative abundance of starting material in the final reaction mixture was a function of the specific peptide and crosslinker, and in some cases none was observed. The three expected Type 2 products were observed in Supplementary Material Figure 1a, namely, $2 / 2(\mathrm{~m} / \mathrm{z} 2106.2,17 \% \mathrm{RA}), 2 / 4(\mathrm{~m} / \mathrm{z}$ 1980.0, $100 \%$ RA), and $4 / 4(\mathrm{~m} / \mathrm{z} 1853.9,47 \% \mathrm{RA})$ crosslinked with DSS. Type 0 products were observed for both 2 $(\mathrm{m} / \mathrm{z} 1140.6,48 \% \mathrm{RA})$ and $4(\mathrm{~m} / \mathrm{z} 1014.5,29 \% \mathrm{RA})$ as well. Products of the reaction between 5 and DSS (Supplementary Material Figure 1b) included Type 1 and Type 0 crosslinked $5(\mathrm{~m} / \mathrm{z}$ 1179.8, 100\% RA and $\mathrm{m} / \mathrm{z} 1197.8,5 \%$ RA). In addition to the expected Type 0,1 , and 2 crosslinked products there were several unexpected species. For example, in Supplementary Material Figure 1a there are species 67 and 97 Da larger than the expected Type 0 products for 4 (at $m / z$ 1081.6, 57\% RA and $m / z 1111.5,9 \%$ RA) and for 2 (at $m / z 1207.7,26 \%$ RA and $m / z 1237.6,12 \%$ RA). These additional products were observed for reactions of DSS, DSG and DSP with 1-4 but not for analogous reactions using DST. Tandem mass spectra of these unexpected products at $+67 \mathrm{Da}$ and $+97 \mathrm{Da}$ does indicate that they are analogs of the expected Type 0 products with the additional mass $(+67 \mathrm{Da},+97 \mathrm{Da})$ localized at the lysine residue (data not shown). The calculated masses of the expected Type 0 products are based on the assumption that the NHSester which does not form a peptide-crosslinker bond will undergo rapid hydrolysis as shown in Scheme 2. If this is not the case and the succinimidyl ester remains intact, the Type 0 product formed will be observed at 97
Da greater in mass. The origin of the +67 Da product is currently unknown; however, given the nitrogen rule, the odd mass shift indicates that the adduct likely contains an odd number of nitrogens (i.e.; at least one). Previous studies of reaction products of DSP revealed impurities in the commercial crosslinker that lead to side reaction products during chemical crosslinking [23]. Possibly in a similar fashion there are amine containing impurities in either the peptide or crosslinker starting materials, which lead to an unexpected crosslink between the peptide and the contaminant. The fact that the adduct is likely to contain at least one nitrogen, and that the adduct was observed localized on the lysine, makes this conclusion plausible. This is one possibility currently being explored. The observation of such abundant side reaction products highlights a potential pitfall when using chemical crosslinkers in protein structure determination. Namely, that unpredicted modifications or reaction products can severely complicate the interpretation of the MS spectra. The chance of a false assignment based solely on precursor $\mathrm{m} / \mathrm{z}$ is increased, and MS/MS confirmation becomes even more important.

For most reactions between the dilysine containing peptides 5 and 6 with a given crosslinker a small amount of high molecular weight product was observed as shown in the Supplementary Material Figure $1 \mathrm{~b}$ inset $(\mathrm{m} / \mathrm{z} 2358.6,0.5 \% \mathrm{RA}$, signal-to-noise of 16.7). In each case, the mass of the observed product is consistent with the type of structure shown in the figure. The proposed structure represents a Type 2,2 product, two peptides intermolecularly crosslinked in two locations. Possibly, this species is actually a gas-phase adduct between two Type 1 crosslinked products. However, for the reactions of 5 and 6 with DST, low abundant species were also observed that would correspond to a Type 2 product $(\mathrm{m} / \mathrm{z}$ 2196.3, a singly intermolecularly crosslinked dimer) and a Type 2,0 product $(\mathrm{m} / \mathrm{z} 2328.3$, a dimer containing one intermolecular crosslink and one dead-end crosslink). These observations support the proposed Type 2,2 structure.

\section{Dissociation of Starting Material}

The MS/MS for each starting material used to generate Type 2 crosslinks are shown in Figure 3. The spectra acquired at identical collision energy values $(\mathrm{CE}=15)$ for the isomers 1, 2, and 3 were qualitatively similar to spectra obtained by tuning the collision energy to deplete each precursor to $5 \%$ of original intensity. The most abundant product ion for peptides 1, 2, and 4 represent dissociation of the peptide bond C-terminal to the lysine residue (i.e.; $\mathrm{y}_{8}, \mathrm{y}_{6}$, and $\mathrm{y}_{8}$, respectively) as shown in Figure $3 a, b$, and d. Preferential cleavage at Lys-Xxx has been previously observed [37]. Peptide 3 (Figure 3c) does not exhibit this cleavage preference, however. The analogous product ion in the case is $y_{2}$ which is observed at only $6 \%$ relative abundance. The most abundant product ions for 3 are $b_{3}(100 \%$ RA) and 

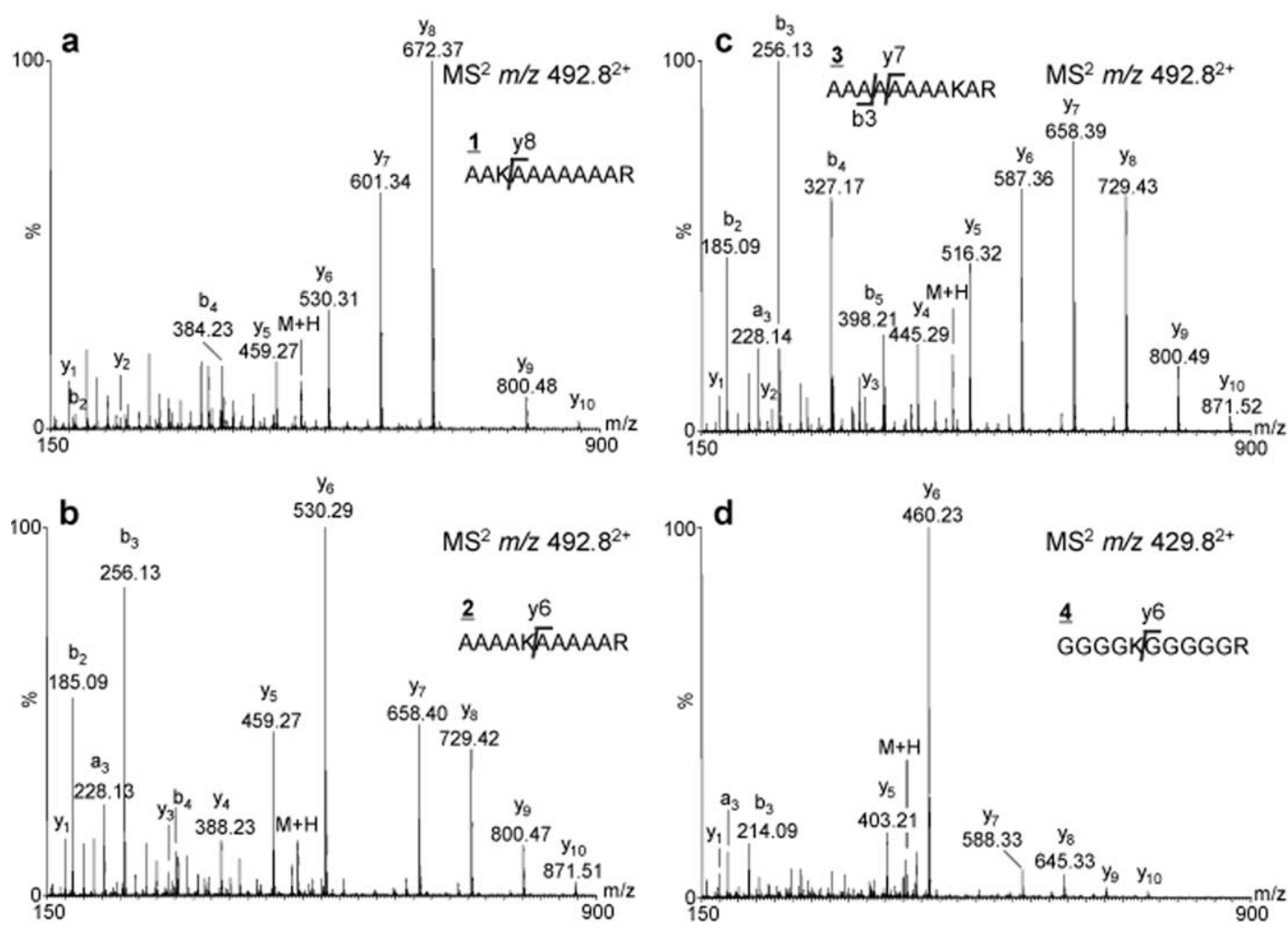

Figure 3. Tandem mass spectra of peptide starting material used in crosslinking reactions to generate Type 2 products.

$\mathrm{y}_{7}(78 \% \mathrm{RA})$, representing dissociation within the polyalanine portion of the molecule. This is most likely observed because a charge driven dissociation to yield a $\mathrm{y}_{2}$ product from the doubly protonated precursor would require both protons to come in close proximity to one another: one proton is expected to be "fixed" at the C-terminal arginine, and a second proton promoting dissociation at Lys2 would thus be only two residues away. Charge repulsion likely prevents this from occurring and promotes greater delocalization of the second proton which drives dissociation within the first few residues of the peptide.

One unexpected observation was the influence of alanine versus glycine on the dissociations observed as seen by comparing products of 2 and 4 (Figure $3 \mathrm{~b}$ and d). In both cases the $y_{6}$ product ion is the base peak in MS/MS, but for the polyglycine peptide this is the only product ion observed above $18 \%$ RA. Alanine is slightly more electron donating than glycine, which may lead to greater proton mobility within the polyalanine backbone and may explain why a greater abundance of dissociation products generated within the polyalanine sequence was observed.

Two peptides (5 and 6 ) each containing 2 Lys residues to promote Type 1 crosslinked products were examined by MS/MS (Supplementary Material Figure 2, (see the electronic version of this article). Peptide 5 with Lys at positions 5 and 7 can be viewed as an analog of 2, while peptide 6 with Lys at positions 3 and 9 is analogous to both 1 and 3. Interestingly, the MS/MS dissociation observed for doubly charged precursor ions of these di-lysine containing peptides 5 and 6 were virtually identical to a monolysine analog, namely 2 and 1, respectively. These observations can be rationalized by considering the most likely sites of protonation for doubly charged 5 and 6 . In each case, the C-terminal arginine will sequester one proton. Two basic sites on each peptide then remain (the two lysine residues). The second proton is more likely to reside on the lysine furthest away from the protonated arginine due to charge repulsion. Thus, for 5 , this second proton is most likely associated with Lys5, which makes the resulting ionic species a direct analog of 2. For peptide 6 the second proton is most likely associated with Lys3, an analogous ion to doubly charged $\mathbf{1}$.

\section{Dissociation of Type 2 Crosslinks: Influence of Precursor Charge State}

Type 2 (intermolecular crosslink) DSS crosslinked peptide 2 was examined by MS/MS in both $2+$ and $3+$ charge states. Given that this species contains two basic residues (arginine), the $2+$ and $3+$ charge states are expected to contain zero and one mobile protons respectively. The deconvoluted MS/MS for each of these species is shown in Figure 4 . The $2+$ precursor requires significantly more energy for dissociation as compared to the $3+$ precursor 

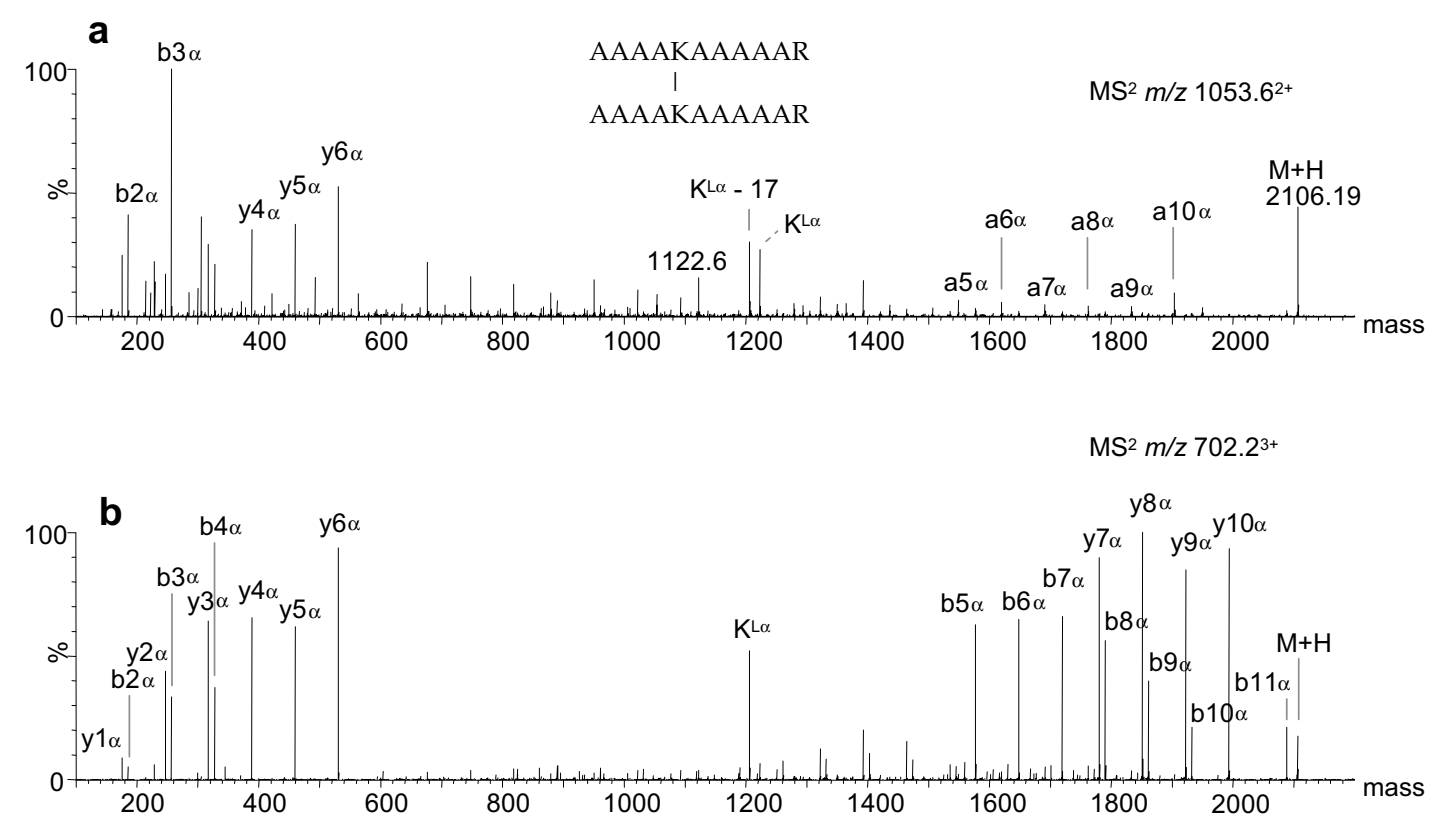

Figure 4. Deconvoluted tandem mass spectra of DSS crosslinked 2. Collision energy was chosen to deplete the precursor ion to $\sim 5 \%$ of the original isolated intensity. (a) $2+$ Precursor MS2 with a collision energy of 50V. (b) $3+$ Precursor MS2 with a collision energy of $12 \mathrm{~V}$.

(50 V versus $12 \mathrm{~V}$ ). In both cases the precursor ion was depleted to $5 \%$ of the initial intensity. The product ions observed for the $2+$ precursor are predominantly low mass species. There are many unique products from the $2+$ precursor observed below $600 \mathrm{Da}$ but these do not represent $\mathrm{a} / \mathrm{b} / \mathrm{y}$ type dissociation products of use for sequence determination. In the 600-1500 Da range the most abundant product ions observed for the $2+$ precursor were also present for the $3+$ precursor although at different relative intensities. Interestingly, the products observed above $1500 \mathrm{Da}$ from the $2+$ precursor were generated by a-type cleavages, rather than the $b / y$ cleavages observed from the $3+$ precursor. Overall, although the $2+$ type 2 crosslinked species dissociates through some unique pathways with respect to the $3+$ analog, it provides fewer and less abundant ions of use for sequence determination. This is similar to results found previously for singly charged isolated peptides-species without a mobile proton. Thus, only $3+$ ions were investigated in the remaining experiments on Type 2 species.

\section{Dissociation of Type 2 Crosslinks: Influence of Crosslinker Identity}

The dissociation pathways open to peptide 2 intermolecularly crosslinked by either DSG, DST, DSS, or DSP were examined to identify and catalogue observed dissociation within the crosslinker moiety and to determine whether the specific crosslinker used had an influence on the product ions observed. B- and y-type cleavages within the peptides were observed for all four precursors examined, and the patterns (relative abun- dance) were qualitatively similar to that shown in Figure $4 \mathrm{~b}$. Thus, for the crosslinkers and model peptide used here, no appreciable difference based on crosslinker identity was observed with respect to dissociation within the peptide. However, product ions arising from cleavage within the crosslinker or apparently promoted by the crosslinker were observed for all four crosslinkers examined. The mass regions of interest for these products are shown in Figure 5a for each product ion spectrum. Deconvoluted spectra are shown for clarity, and the mass regions are shown relative to each other such that analogous dissociation products are vertically aligned. Assignments for the observed dissociations are pictured in Figure $5 \mathrm{~b}$ and are discussed below.

Products from two dissociation pathways internal to the crosslinker were observed. The first involves cleavage within the amide bond formed between crosslinker and lysine residue which was observed for all four crosslinkers used in this study. This dissociation is analogous to $b$ - and y-type cleavage within the peptide backbone and has been cited previously [25, 41]. Two product ions may be formed as shown in Figure $5 b$ labeled as $\mathrm{L} \alpha 5$ and $\mathrm{L} \beta 13$ according to previously proposed nomenclature [25]. The former (L $\alpha 5)$ represents a y-like dissociation and would appear at $\mathrm{m} / z 984.6^{+}$for each of the four precursors examined because this product ion essentially corresponds to peptide 2 . However, this product could only definitively be observed for DSG crosslinked 2 due to overlapping components within this mass region for the DST, DSS, and DSP crosslinked species (data not shown). The other product 

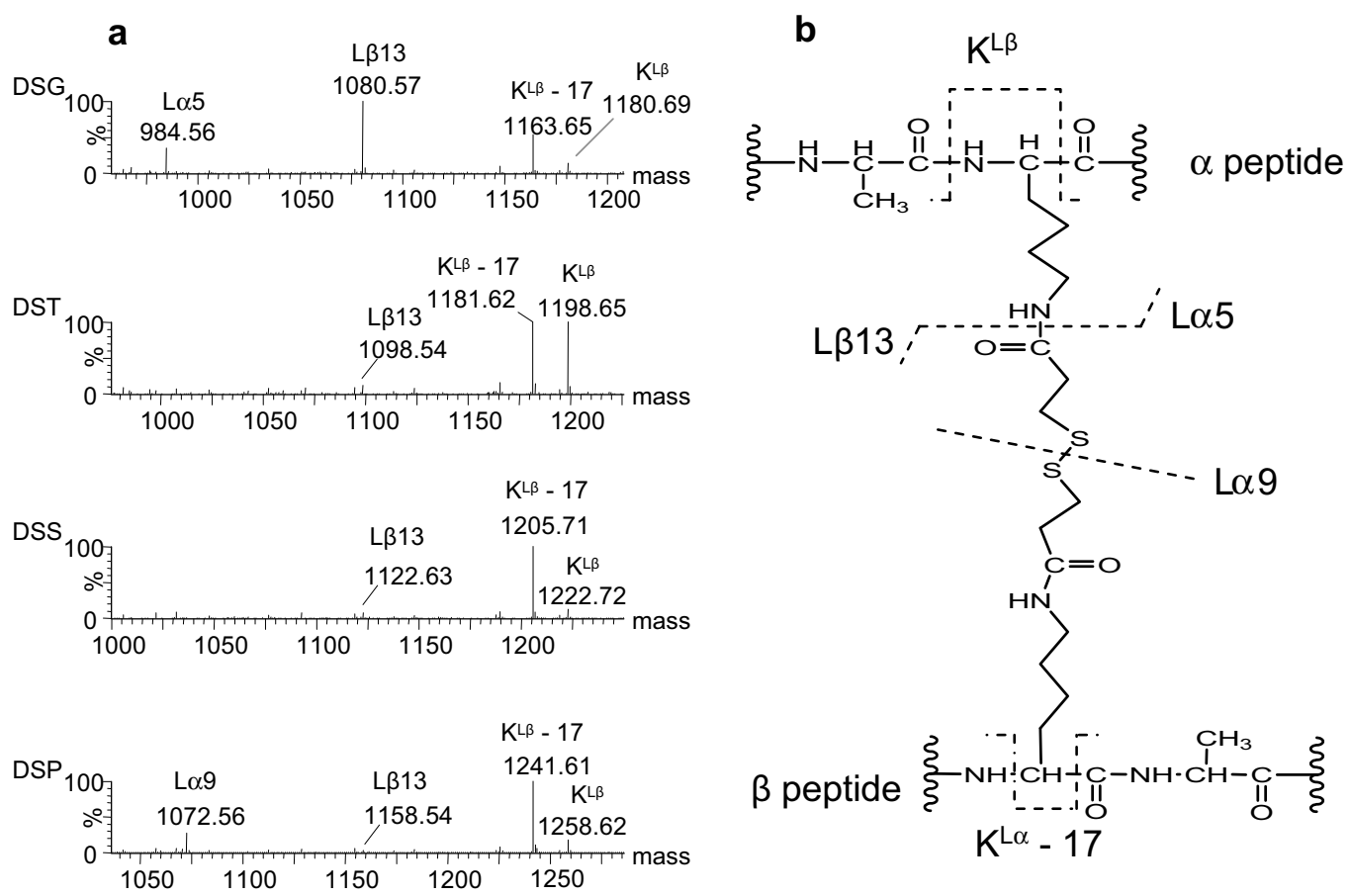

peptide

$$
\mathrm{K}^{\mathrm{L} \alpha}-17
$$

Figure 5. (a) Selected regions of MS2 spectra from DSG, DST, DSS, and DSP crosslinked 2 showing dissociation products within or promoted by the crosslinker. Deconvoluted spectra are shown for clarity, and mass regions are shown relative to each other such that analogs dissociation products are vertically aligned. (b) Assignments for the dissociation products from (a). The DSP crosslinked precursor is shown with nomenclature based on reference [25]. Nomenclature for labeling the $\mathrm{K}$ immonium ion is proposed in the text of the current manuscript. Note that in the example shown $\mathrm{K}^{\mathrm{L} \alpha}$, $\mathrm{K}^{\mathrm{L} \alpha}-17$, and $\mathrm{L} \alpha 9$ are equivalent to $\mathrm{K}^{\mathrm{L} \beta}, \mathrm{K}^{\mathrm{L} \beta}-17$, and $\mathrm{L} \beta 9$, respectively, due to symmetry within the molecule.

(L $\beta 13)$ results from a b-like dissociation and includes the crosslinker moiety. DSG crosslinked 2 yielded the highest abundance of this product relative to the other precursors, which was observed at both $\mathrm{m} / \mathrm{z} 1080.6^{+}$ and $m / z 540.8^{2+}$ in the original spectrum. The DST, DSS, and DSP crosslinked precursors yielded a minor amount of this b-like dissociation product at $\mathrm{m} / \mathrm{z}$ $1098.5^{+}, \mathrm{m} / \mathrm{z} 1122.6^{+}$, and $\mathrm{m} / \mathrm{z} 1158.5^{+}$, respectively. No appreciable amount of the corresponding doubly charged species were observed.

The second dissociation observed within a crosslinker was unique to DSP and represented dissociation of the disulfide bond present in this crosslinker. As shown in Figure $5 b$, dissociation of the $\mathrm{S}-\mathrm{S}$ bond occurs with proton transfer to the resulting observed product ion, presumably forming a sulfhydryl moiety. This product is observed at $\mathrm{m} / \mathrm{z} 1072.6$ (Figure 5a).

In addition to dissociation within the crosslinker moiety, the presence of a crosslinker in these Type 2 species appears to promote particular two-bond cleavages within the peptide backbone. The first series of products appears for DSG, DST, DSS, and DSP crosslinked 2 at $\mathrm{m} / \mathrm{z} 1180.69^{+}, 1198.65^{+}, 1222.72^{+}$and $1258.62^{+}$, respectively, while the second series appears 17 Da lower in mass (Figure 5a). The dissociating bonds are directly adjacent to the site of crosslinking in each case as diagrammed in Figure $5 b$. The first series essentially represents the formation of the lysine immonium ion with the crosslinker and second peptide "modification" still attached. Thus, we propose to annotate this product as $\mathrm{K}^{\mathrm{L} \beta}$ where the subscripts $\mathrm{L}$ and $\beta$ represent "linker" and " $\beta$ peptide", respectively. The second series is likely a loss of ammonia from the first series. Interestingly, previous researchers annotated similar dissociation pathways in crosslinked species they examined although it was not the focus of their investigation [34]. In addition, Schilling and coworkers [25] observed an equivalent cleavage product for type 0 crosslinked species $\left(\mathrm{K}^{\mathrm{L}}-17\right)$ and proposed a mechanism to account for the facile loss of ammonia. For the DST crosslinked species examined in the present work, the $\mathrm{K}^{\mathrm{L} \beta}$ product was as abundant as $\mathrm{K}^{\mathrm{L} \beta}-17$. A small amount of the $\mathrm{K}^{\mathrm{L} \beta}$ product ion was observed for DSG, DSS, and DSP crosslinked species but the corresponding $\mathrm{K}^{\mathrm{L} \beta}-17$ product was observed at greater abundance. Note that in this case, because the $\alpha$ and $\beta$ peptides are equivalent, these products could be annotated equivalently as $\mathrm{K}^{\mathrm{L} \alpha}$ or $\mathrm{K}^{\mathrm{L} \beta}$. Ongoing work in our laboratory [42] suggests that these $\mathrm{K}^{\mathrm{L} \alpha} / \mathrm{K}^{\mathrm{L} \beta}$ product ions are present even when the crosslinked peptides contain amino acids such as aspartic acid, D, known to itself promote dissociation of the peptide backbone. 


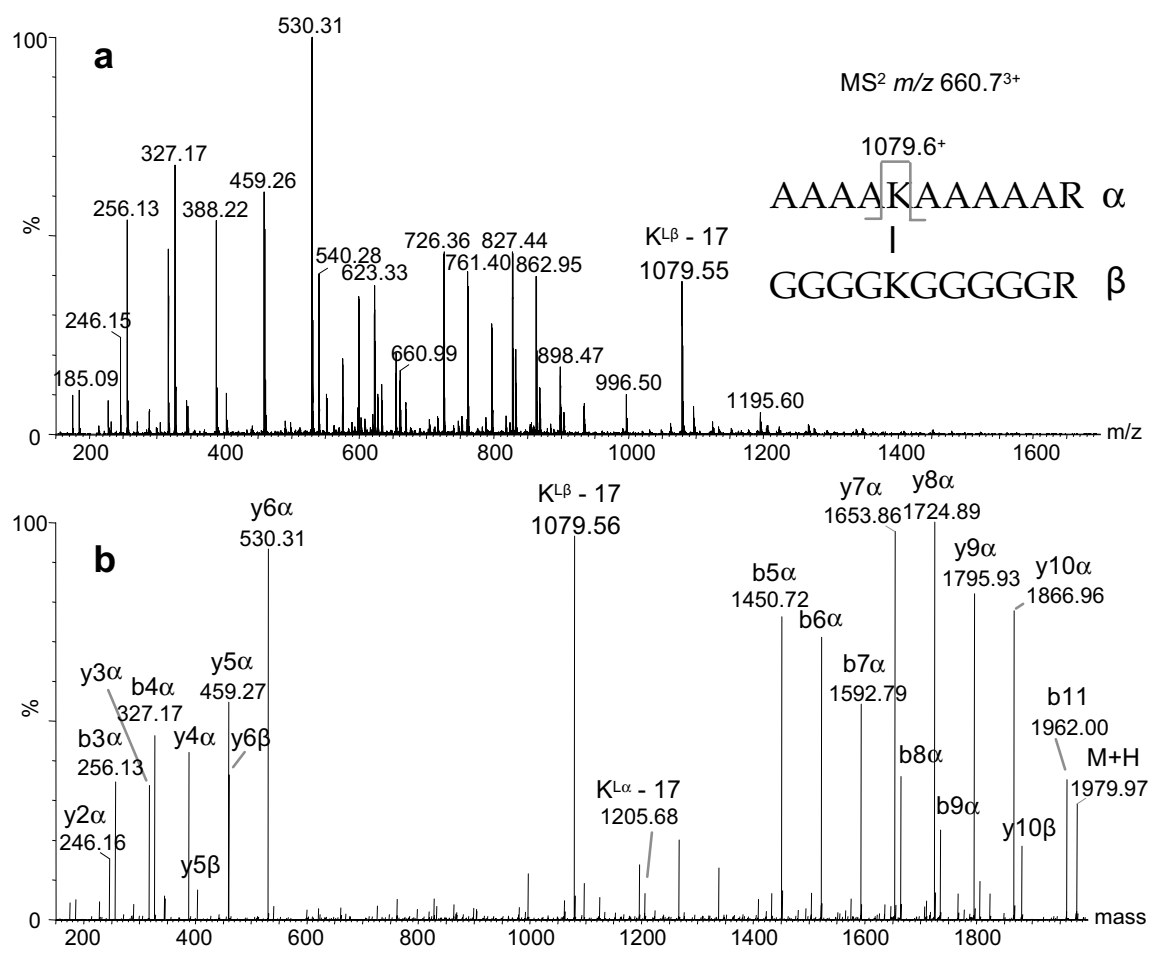

Figure 6. Tandem mass spectrum of the hetero Type 2 DSS crosslinked peptides 2 ( $\alpha$ chain) and 4 ( $\beta$ chain), (a) original MS2 data, (b) spectrum deconvoluted with MaxEnt3.

\section{Dissociation of Type 2 Crosslinks: Influence of Crosslink Location.}

Dissociation patterns for Type 2 crosslinked polyalanine peptides were examined as a function of crosslink location relative to the peptide $C$ terminus. Triply charged precursors for the DSS-crosslinked species of peptides 1, 2, and 3 (homo-crosslinked products) were examined. Collision energy for each precursor was $12 \mathrm{~V}$ which also served to deplete each precursor ion to $5 \%$ of the original intensity. The primary difference among the three spectra, shown in Supplementary Material Figure 3, (which can be found in the electronic version of this article.) was the charge state distribution of the product ions formed. No ( $<3 \%$ RA) $3+$ charged products were formed for DSS crosslinked 3 (Supplementary Material Figure 3c), where the site of crosslinking was located near the $\mathrm{C}$ terminus. For MS/MS of DSS crosslinked 1 and 2 however (Supplementary Material Figure $3 a$ and $b$ ), the $y$ ion series appeared as $3+$ species in addition to the $2+$ species observed for all three precursors. Similarly the $\mathrm{K}^{\mathrm{L} \beta}-17$ cleavage product ion discussed above was observed in a $2+$ charge state at $\mathrm{m} / \mathrm{z} 603.3^{2+}$ for crosslinked 1 and 2 but only in a $1+$ charge state $\left(\mathrm{m} / \mathrm{z} 1205.7^{+}\right)$for crosslinked 3 . In fact, this product ion was observed only as a $2+$ ion for crosslinked 1; no 1+ charge state was observed for this precursor, where the site of crosslinking was close to the N-terminus.

The dissociation patterns of hetero-crosslinked species were also examined as a function of crosslink location. Polyglycine peptide 4 was DSS-crosslinked with one of the polyalanine peptides 1,2 , or 3 and the resulting $3+$ species was allowed to undergo collision induced dissociation such that each precursor ion was depleted to $5 \%$ of the initial signal intensity. The type and charge state distribution of the observed products were qualitatively similar to the corresponding homocrosslinked products of 1,2, and 3. Dissociation of the peptide 4 moiety was minimal to absent in each heterocrosslinked species examined. Most interesting, there was a distinct preference for dissociation within the polyalanine peptide and not the polyglycine peptide such that $K^{\mathrm{L} \beta}-17(\mathrm{~m} / \mathrm{z} 1079.6)$ and not $\mathrm{K}^{\mathrm{L} \alpha}-17(\mathrm{~m} / \mathrm{z}$ 1205.7) was observed preferentially.

The MS/MS spectrum acquired for the $4 / 2$ crosslinked species is shown in Figure 6 (original and deconvoluted). Comparison of the spectrum in Figure 6a and Supplementary Material Figure $3 \mathrm{~b}$ is representative of the close correspondence observed between the homo-crosslinked and analogous hetero-crosslinked species. For example, the relative abundance ratio of singly to doubly charged $\mathrm{K}^{\mathrm{L} \beta}-17$ product is $\sim 1$ in each case: 0.954 for the hetero-crosslinked species and 1.016 for the homocrosslinked species. For the $4 / 3$ crosslinked species (data not shown) no $(<3 \%$ RA) $3+$ y ions were observed, and $\mathrm{K}^{\mathrm{L} \beta}-17$ was only observed at $\mathrm{m} / \mathrm{z} 1079.6^{+}$as a singly charged species similar to the $3 / 3$ crosslinked species. The 4/1 crosslinked species (data not shown, but similar to $1 / 1$ ) yielded both $2+$ and $3+y$ ions as well as predominantly $2+$ products for $\mathrm{K}^{\mathrm{L} \beta}-17$. 


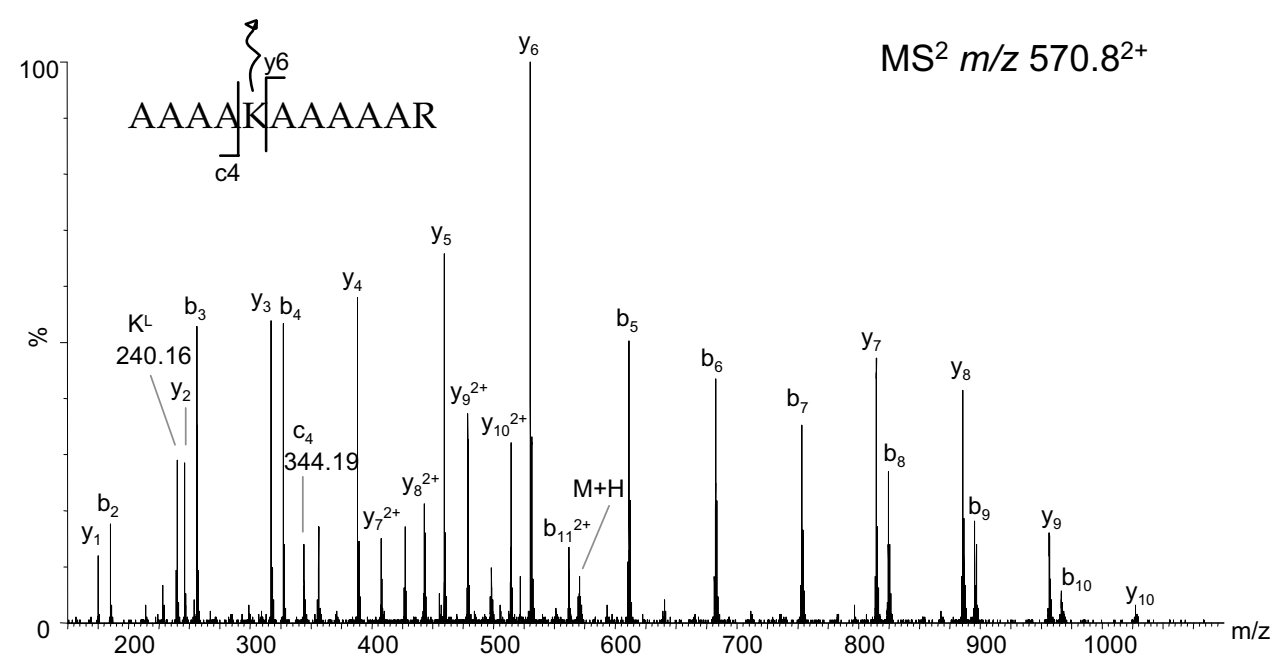

Figure 7. Tandem mass spectrum of the Type 0 species formed between DSS and 2.

\section{Dissociation of Type 0 Species}

The NHS esters used for these crosslinking reactions hydrolyze over time in the presence of water as shown in Scheme 2. If hydrolysis of the remaining NHS ester occurs after the first reacts with a primary amine on a peptide, the intra or intermolecular crosslink can no longer be formed. Thus, a common side reaction product formed during chemical crosslinking with NHS esters is the so called "dangling" crosslink, or Type 0 species. Type 0 species formed in the reactions between peptide 1, 2, or 3 with DSS and in reactions between peptide 2 with DSG, DST, and DSP were examined to determine the similarities and differences in dissociation pathways observed as compared to analogous starting material or Type 2 species.

The CID spectrum acquired for the DSS/ 2 Type 0 species generated in this study is shown in Figure 7. The base peak in this spectrum arises from $\mathrm{y}_{6}$ cleavage, similar to what is observed for the unmodified peptide (see Figure 3). In contrast to the unmodified peptide, however, the relative intensity of certain y ions, particularly $y_{2}, y_{3}$, and $y_{4}$ are significantly increased in the Type 0 species, and nearly the entire y ion series is observed. In addition, the entire $b$ ion series is observed $\left(b_{2}-b_{10}\right)$ for the Type 0 species, whereas only $b_{2}-b_{4}$ were observed above $10 \%$ RA for the unmodified peptide 2 . The presence of nearly the entire series of $b$ and $y$ ion products $\left(b_{3}-b_{10}\right.$ and $\left.y_{2}-y_{11}\right)$ was similarly observed for all Type 0 species examined. Thus, this observation was independent of crosslinker type or position. The less selective cleavage observed for the Type 0 species is mostly likely because in the Type 0 species, the only strongly basic site is the C-terminal arginine. Thus, for these doubly charged Type 0 precursors, one proton is sequestered at $R$ and the second proton is relatively freer to drive dissociation at any point along the remainder of the peptide backbone than for the unmodified peptide containing an internal lysine. This is also typical of the product distribution for triply charged Type 2 species examined in this study.

No products were observed to arise solely from the Type 0 precursor of a given crosslinker (DSS, DSG, DST, DSP). Products corresponding to the S-S bond dissociation (expected at $\mathrm{m} / \mathrm{z} 1072.6$ or 107.0) for DSP were not observed. Also, products corresponding to dissociation of the amide bond between crosslinker and peptide were not observed. Products arising from the two bond $\mathrm{K}^{\mathrm{L}}$ cleavage were observed for the Type 0 species of DSG $(\mathrm{m} / \mathrm{z} 198.11)$, DST $(\mathrm{m} / z$ 216.08), DSS $(\mathrm{m} / \mathrm{z} 240.16)$, and DSP $(m / z 276.08)$. In addition, the product of single bond dissociation at $\mathrm{c}_{4}$ is observed for the DSG, DST, DSS, and DSP crosslinked peptide 2 at $m / z 344.2^{+}$. Note that this represents dissociation of the bond immediately adjacent to the site of crosslinking. The c cleavage is not observed for unmodified peptide 2 suggesting that the presence of the crosslinker modification promotes this dissociation process. The analogous $c_{2}$ or $c_{8}$ single bond dissociation products that might be expected from Type 0 modified 1 or 3 are not observed, however.

\section{Dissociation of Type 1 Crosslinks}

The singly and doubly charged precursors for the Type 1 (intramolecular) crosslinked peptide 5 with DSS were examined by MS/MS. In a manner similar to the Type 2 species examined, Type 1 peptides lacking a mobile proton $(1+$ species) required significantly more energy for dissociation than the doubly charged precursor $(65$ versus $12 \mathrm{~V}$, respectively). Additionally, the product ions generated from the singly charged precursor are relatively low molecular weight species with few $b$ or $y$ ions useful for deducing sequence.

Tandem mass spectrometric dissociation of doubly charged Type 1 crosslinked peptides were then examined as a function of distance between the sites of 


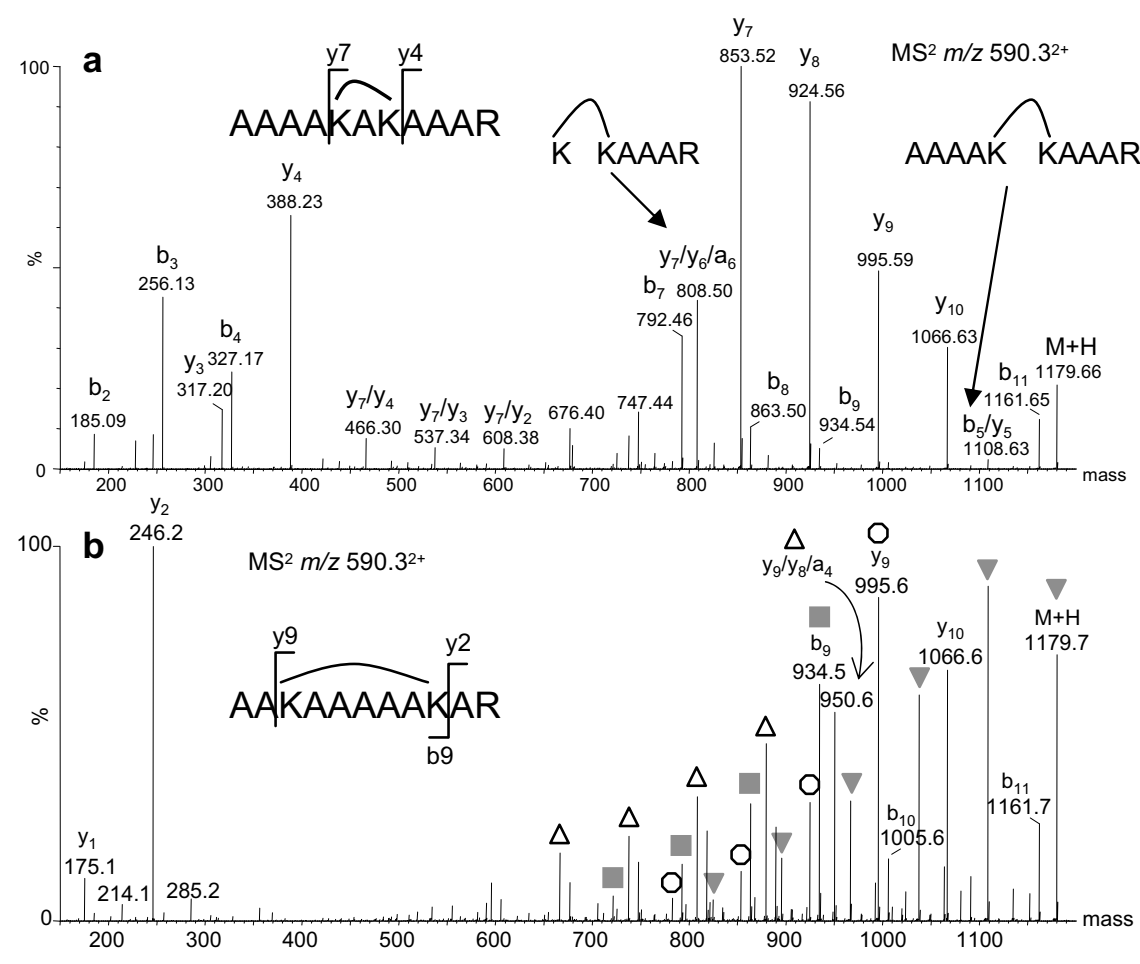

Figure 8. (a) Deconvoluted tandem mass spectrum of Type 1 DSS crosslinked 5. (b) Deconvoluted tandem mass spectrum of Type 1 DSS crosslinked 6 . Each series of equivalent markings (e.g.; filled triangle, filled square, open circle, and open triangle) indicates the loss of $\mathrm{Ala}_{\mathrm{n}}$ (where $n=1$ up to 5) from the "parent" structure (e.g.; $\mathrm{M}+\mathrm{H}, \mathrm{b} 9, \mathrm{y} 9, \mathrm{y} 9 / \mathrm{y} 8 / \mathrm{a} 4$ ) within the Ala4-Ala8 sequence.

crosslinking by comparing MS/MS of DSS crosslinked 5 (Figure $8 \mathrm{a}$ ) versus 6 (Figure $8 \mathrm{~b}$ ). The tandem mass spectra of Type 1 species are substantially more complex than the Type 2 species examined, particularly as the distance between the 2 sites of intra-molecular crosslinking increases.

It is noteworthy that intramolecularly crosslinked peptides may be viewed as cyclic peptide analogs. More specifically, Type 1 crosslinked 5 and 6 are analogous to species containing both cyclic and linear peptide moieties. Based on previous tandem mass spectrometry studies of cyclic peptides [43], it is therefore likely that any of the peptide bonds between Ala4 and Ala8 in Type 1 crosslinked 6 undergo a "ring opening" dissociation. The resulting ion now has two additional termini from which dissociation of these amino acids may occur. In the case of crosslinked 5 a ring opening dissociation is restricted to the bonds on either side of Ala6, and from there only one additional terminus is formed, making the origin of observed products unambiguous. Because 6 is based on polyalanine, many of the possible dissociation products would have identical $\mathrm{m} / \mathrm{z}$ values making them indistinguishable. For example, the product ion labeled as originating from a $\mathrm{y}_{9} / \mathrm{y}_{8} / \mathrm{a}_{4}$ cleavage could also have arisen from $y_{9} / y_{7} / a_{3}$. This suggests that MS/MS of a more heterogeneous Type 1 crosslinked peptide, a subject of future studies, may be much more complex. In that case, multiple stages of mass spectrometry may be required to sequence such species as has been proposed for cyclic peptides [43].
Thus, Type 1 crosslinked 5 has only a few additional product ions in comparison to the unmodified starting material. In particular, the products resulting from $b_{5} / y_{5}$ and $y_{7} / y_{6} / a_{6}$ multiple bond dissociations are observed. As shown in Figure 8a, these products involve the loss of Ala6 from the precursor ion. In contrast, the MS/MS for Type 1 crosslinked 6 is very complex due to so many additional product ions as compared to both the unmodified peptide 6 and crosslinked 5. This is illustrated in Figure $8 \mathrm{~b}$, where successive losses of $\mathrm{Ala}_{\mathrm{n}}$ from the precursor or in conjunction with other dissociations (for example, $\mathrm{y}_{9}$ or $b_{9}$ ) are annotated with identical markers (e.g.; shaded triangle, shaded square, open circle, and open triangle.)

Another prominent feature of these Type 1 precursors is the y cleavages at each site of crosslinking are greatly enhanced in comparison with that observed for the unmodified starting material. Thus, cleavages at $\mathrm{y}_{4}$ and $y_{7}$ are prominent in the MS/MS of Type 2 crosslinked 5, while those at $\mathrm{y}_{2}$ and $\mathrm{y}_{9}$ are prominent in the MS/MS of 6 . Type 1 species of DSG, DST and DSP crosslinked 5 and $\mathbf{6}$ were also examined by MS/MS, however, no differences in these spectra were observed with respect to the DSS crosslinked analog.

\section{Conclusions}

Tandem mass spectra for Type 0,1 , and 2 crosslinked species (dangling, intramolecular and intermolecular crosslinks) were examined. A series of crosslinkers and 
peptides was used to probe the effect of precursor charge state, crosslinker identity and crosslink type/ location on the resulting product distribution. Similar to unmodified peptides, precursor ions lacking a mobile proton required more energy for dissociation and yielded fewer ions for sequence determination than precursors with a mobile proton. The specific crosslinker used (DSG, DST, DSS or DSP) did not promote differential cleavage within the peptide moiety in the Type 0,1 , or 2 precursors examined. Dissociation of the peptide bonds adjacent to the site of crosslinking was enhanced, however, independent of the crosslinker used. For Type 2 precursors, dissociation within the crosslinker was also observed at the amide bond between peptide and crosslinker. One crosslinker-specific dissociation product was observed corresponding to $\mathrm{S}-\mathrm{S}$ bond dissociation within the crosslinker DSP.

Crosslink location influenced the dissociation products observed in two ways. For the Type 2 precursors examined, site of crosslinking influenced the charge state of MS/MS products observed. Crosslinking near the C-terminus yielded products in lower charges than analogous products from precursors crosslinked near the $\mathrm{N}$-terminus. For the Type 1 precursors examined, greater distance between the crosslinker sites of attachment lead to a greater number of internal cleavage products observed and, thus, more complex MS/MS data.

Observed trends in the dissociation patterns obtained for these species will allow for improvements to software used in the automated interpretation of crosslinked peptide MS/MS.

\section{Acknowledgments}

The authors acknowledge the United States Department of Energy Office of Science for funding through the Genomics:GTL program. SPG thanks Rick Jacobsen and Drs. Michael Leavell and Gregory Hurst for helpful discussions. SPG also thanks Tahera Iqbal for critical reading of the manuscript. Sandia is a multiprogram laboratory operated by Sandia Corporation, a Lockheed Martin Company, for the United States Department of Energy's National Nuclear Security Administration under contract DE-AC0494AL85000.

\section{References}

1. Livney, Y. D.; Schwan, A. L.; Dalgleish, D. G. A Study of $\beta$-Casein Tertiary Structure by Intramolecular Crosslinking and Mass Spectrometry. J. Dairy Sci. 2004, 87(11), 3638-3647.

2. Kruppa, G.; Schoeniger, J.; Young, M. A Top Down Approach to Protein Structural Studies Using Chemical Cross-Linking and Fourier Transform Mass Spectrometry. Rapid Commun. Mass Spectrom. 2003, 17(2), $155-162$.

3. Back, J. W.; de Jong, L.; Muijsers, A. O.; de Koster, C. G. Chemical Cross-Linking and Mass Spectrometry for Protein Structural Modeling. J. Mol. Biol. 2003, 331(2), 303-313.

4. Taverner, T.; Hall, N.; O'Hair, R.; Simpson, R. Characterization of an Antagonist Interleukin-6 Dimer by Stable Isotope Labeling, CrossLinking, and Mass Spectrometry. J. Biol. Chem. 2002, 277(48), 4648746492.

5. Pearson, K.; Pannell, L.; Fales, H. Intramolecular Cross-Linking Experiments on Cytochrome $c$ and Ribonuclease $a$ Using an Isotope Multiplet Method. Rapid Commun. Mass Spectrom. 2002, 16(3), 149-159.
6. Kim, H.; Foster, M. Characterization of Ad5 E3-14.7k, an Adenoviral Inhibitor of Apoptosis: Structure, Oligomeric State, and Metal Binding. Protein Sci. 2002, 11(5), 1117-1128.

7. Young, M. M.; Tang, N.; Hempel, J. C.; Oshiro, C. M.; Taylor, E. W.; Kuntz, I. D.; Gibson, B. W.; Dollinger, G. High Throughput Protein Fold Identification by Using Experimental Constraints Derived from Intramolecular Cross-Links and Mass Spectrometry. Proc. Natl. Acad. Sci. U.S.A. 2000, 97(11), 5802-5806.

8. Wu, Y.; Engen, J. R. What Mass Spectrometry Can Reveal About Protein Function. Analyst 2004, 129(4), 290-296.

9. Dziembowski, A.; Seraphin, B. Recent Developments in the Analysis of Protein Complexes. FEBS Lett. 2004, 556(1/3), 1-6.

10. Back, J.; Sanz, M.; De Jong, L.; De Koning, L.; Nijtmans, L.; De Koster, C.; Grivell, L.; Van der Spek, H.; Muijsers, A. A Structure for the Yeast Prohibitin Complex: Structure Prediction and Evidence from Chemical Crosslinking and Mass Spectrometry. Protein Sci. 2002, 11(10), 24712478.

11. Rappsilber, J.; Siniossoglou, S.; Hurt, E.; Mann, M. A Generic Strategy to Analyze the Spatial Organization of Multi-Protein Complexes by CrossLinking and Mass Spectrometry. Anal. Chem. 2000, 72(2), 267-275.

12. Venkataraman, G.; Shriver, Z.; Davis, J.; Sasisekharan, R. Fibroblast Growth Factors 1 and 2 are Distinct in Oligomerization in the Presence of Heparin-Like Glycosaminoglycans. Proc. Natl. Acad. Sci. U.S.A. 1999, 96(5), 1892-1897.

13. Farmer, T.; Caprioli, R. Determination of Protein-Protein Interactions by Matrix-Assisted Laser Desorption/Ionization Mass Spectrometry. J. Mass Spectrom. 1998, 33(8), 697-704.

14. Vater, J.; Heinze, K.; Friedrich, B.; Kablitz, B.; Blokesch, A.; Irrgang, K.; Thiede, B.; Salnikow, J. Novel Methodology for Topological Studies of Photosynthetic Membrane Protein Complexes. Ber. Bunsen-Ges. Phys. Chem 1996, 100(12), 2107-2111.

15. Kaufmann, R.; Kirsch, D.; Tourmann, J.; Machold, J.; Hucho, F.; Utkin, Y.; Tsetlin, V. Matrix-Assisted Laser-Desorption Ionization (MALDI) and Post-Source Decay (PSD) Product Ion Mass Analysis Localize a Photolabel Cross-Linked to the $\delta$-Subunit of Nachr Protein by Neurotoxin-Ii. Eur. Mass Spectrom. 1995, 1(3), 313-325.

16. Chu, F. X.; Shan, S. O.; Moustakas, D. T.; Alber, F.; Egea, P. F.; Stroud, R. M.; Walter, P.; Burlingame, A. L. Unraveling the Interface of Signal Recognition Particle and Its Receptor by Using Chemical Cross-Linking and Tandem Mass Spectrometry. Proc. Natl. Acad. Sci. U.S.A. 2004, 101(47), 16454-16459.

17. Lanman, J.; Lam, T.; Barnes, S.; Sakalian, M.; Emmett, M.; Marshall, A.; Prevelige, P. Identification of Novel Interactions in Hiv-1 Capsid Protein Assembly by High-Resolution Mass Spectrometry. J. Mol. Biol. 2003, 325(4), 759-772.

18. Zaitseva, L.; Ovchinnikova, T.; Vodovozova, E.; Molotkovsky, Y.; Polyakov, N.; Titov, M.; Esipov, S.; Grinkevich, V. Mitochondrial H+-Atpase: Identification of Subunits of the F-0 Subcomplex That Contact Membrane Lipids. Russ. J. Bioorg. Chem. 2002, 28(5), 371-384.

19. A Two-Tracked Approach to Analyze RNA-Protein Crosslinking Sites in Native, Nonlabeled Small Nuclear Ribonucleoprotein Particles. Methods 2002, 26(2), 170-181.

20. Bennett, K.; Kussmann, M.; Bjork, P.; Godzwon, M.; Mikkelsen, M.; Sorensen, P.; Roepstorff, P. Chemical Cross-Linking with ThiolCleavable Reagents Combined with Differential Mass Spectrometric Peptide Mapping-A Novel Approach to Assess Intermolecular Protein Contacts. Protein Sci. 2000, 9(8), 1503-1518.

21. Alloza, I.; Martens, E.; Hawthorne, S.; Vandenbroeck, K. Cross-Linking Approach to Affinity Capture of Protein Complexes from ChaotropeSolubilized Cell Lysates. Anal. Biochem. 2004, 324(1), 137-142.

22. Rashid, K.; Hevi, S.; Chen, Y.; Le Caherec, F.; Chuck, S. A Proteomic Approach Identifies Proteins in Hepatocytes That Bind Nascent Apolipoprotein B. J. Biol. Chem. 2002, 277(24), 22010-22017.

23. Swaim, C. L.; Smith, J. B.; Smith, D. L. Unexpected Products from the Reaction of the Synthetic Cross-Linker 3,3'-Dithiobis(Sulfosuccinimidyl Propionate), DTSSP with Peptides. J. Am. Soc. Mass Spectrom. 2004, 15(5), 736-749.

24. Leavell, M. D.; Novak, P.; Behrens, C. R.; Schoeniger, J. S.; Kruppa, G. H. Strategy for Selective Chemical Cross-Linking of Tyrosine and Lysine Residues. J. Am. Soc. Mass Spectrom. 2004, 15(11), 1604-1611.

25. Schilling, B.; Row, R.; Gibson, B.; Guo, X.; Young, M. Ms2assign, Automated Assignment and Nomenclature of Tandem Mass Spectra of Chemically Crosslinked Peptides. J. Am. Soc. Mass Spectrom. 2003, 14(8), 834-850.

26. Craig, R.; Krokhin, O.; Wilkins, J.; Beavis, R. Implementation of an Algorithm for Modeling Disulfide Bond Patterns Using Mass Spectrometry. J. Proteome Res. 2003, 2(6), 657-661.

27. Jahn, O.; Hofmann, B.; Brauns, O.; Spiess, J.; Eckart, K. The Use of Multiple Ion Chromatograms in on-Line HPLC-MS for the Characterization of Post-Translational and Chemical Modifications of Proteins. Int. J. Mass Spectrom. 2002, 214(1), 37-51.

28. Chen, T.; Jaffe, J. D.; Church, G. M. Algorithms for Identifying Protein Cross-Links via Tandem Mass Spectrometry. J. Comput. Biol. 2001, 8(6), 571-583.

29. Hurst, G. B.; Lankford, T. K.; Kennel, S. J. Mass Spectrometric Detection of Affinity Purified Crosslinked Peptides. J. Am. Soc. Mass Spectrom. 2004, 15(6), 832-839.

30. Fujii, N.; Jacobsen, R.; Wood, N.; Schoeniger, J.; Guy, R. A Novel Protein Crosslinking Reagent for the Determination of Moderate Resolution 
Protein Structures by Mass Spectrometry (Ms3-D). Bioorg. Med. Chem. Lett. 2004, 14(2), 427-429.

31. Denison, C.; Kodadek, T. Toward a General Chemical Method for Rapidly Mapping Multi-Protein Complexes. J. Proteome Res. 2004, 3(3), $417-425$.

32. Collins, C.; Schilling, B.; Young, M.; Dollinger, G.; Guy, R. Isotopically Labeled Crosslinking Reagents: Resolution of Mass Degeneracy in the Identification of Crosslinked Peptides. Bioorg. Med. Chem. Lett. 2003, 13(22), 4023-4026.

33. Back, J.; Hartog, A.; Dekker, H.; Muijsers, A.; de Koning, L.; de Jong, L. A New Crosslinker for Mass Spectrometric Analysis of the Quaternary Structure of Protein Complexes. J. Am. Soc. Mass Spectrom. 2001, 12(2), 222-227.

34. Chen, X.; Chen, Y.; Anderson, V.; Protein Cross-Links: Universal Isolation and Characterization by Isotopic Derivatization and Electrospray Ionization Mass Spectrometry. Anal. Biochem. 1999, 273(2), 192203.

35. Tsaprailis, G.; Somogyi, A.; Nikolaev, E. N.; Wysocki, V. H. Refining the Model for Selective Cleavage at Acidic Residues in Arginine-Containing Protonated Peptides. Int. J. Mass Spectrom. 2000, 196, 467-479.

36. Huang, Y.; Wysocki, V. H.; Tabb, D. L.; Yates, J. R. III. The Influence of Histidine on Cleavage C-Terminal to Acidic Residues in Doubly Protonated Tryptic Peptides. Int. J. Mass Spectrom. 2002, 219(1), 233-244.
37. Kish, M. M.; Wesdemiotis, C. Selective Cleavage at Internal Lysine Residues in Protonated vs. Metalated Peptides. Int. J. Mass Spectrom. 2003, 227(1), 191-203.

38. Breci, L. A.; Tabb, D. L.; Yates, J. R.; Wysocki, V. H. Cleavage N-Terminal to Proline: Analysis of a Database of Peptide Tandem Mass Spectra. Anal. Chem. 2003, 75(9), 1963-1971.

39. Tabb, D. L.; Huang, Y. Y.; Wysocki, V. H.; Yates, J. R. Influence of Basic Residue Content on Fragment Ion Peak Intensities in Low-EnergyCollision-Induced Dissociation Spectra of Peptides. Anal. Chem. 2004, $76(5), 1243-1248$.

40. Tsaprailis, G.; Nair, H.; Zhong, W.; Kuppannan, K.; Futrell, J. H.; Wysocki, V. H. A Mechanistic Investigation of the Enhanced Cleavage at Histidine in the Gas-Phase Dissociation of Protonated Peptides. Anal. Chem. 2004, 76(7), 2083-2094.

41. Raftery, M.; Geczy, C.; Electrospray Low Energy CID and Maldi PSD Fragmentations of Protonated Sulfinamide Cross-Linked Peptides. J. Am. Soc. Mass Spectrom. 2002, 13(6), 709-718.

42. Gaucher, S. P.; Hadi, M. Z.; Young, M. M. Investigation of Gas-Phase Dissociation Pathways of Lys-Lys Crosslinked Peptides: Influence of Crosslinker Identity and Position. Proceedings of the 53rd ASMS Conference on Mass Spectrometry; San Antonio, TX, June, 2005.

43. Ngoka, L. C. M.; Gross, M. L. Multistep Tandem Mass Spectrometry for Sequencing Cyclic Peptides in an Ion-Trap Mass Spectrometer. J. Am. Soc. Mass Spectrom. 1999, 10(8), 732-746. 